\title{
A Two-Step Identification Approach for Twin-Box Models of RF Power Amplifier
}

\author{
You-Jiang Liu, ${ }^{1,2}$ Bang-Hua Zhou, ${ }^{2}$ Jie Zhou, ${ }^{2}$ and Yi-Nong Liu' \\ ${ }^{1}$ Department of Engineering Physics, Tsinghua University, Beijing 100084, China \\ ${ }^{2}$ Institute of Electronic Engineering, China Academy of Engineering Physics, Mianyang 621900, China
}

Correspondence should be addressed to You-Jiang Liu, goowuu@gmail.com

Received 21 April 2011; Revised 11 July 2011; Accepted 19 July 2011

Academic Editor: Paolo Colantonio

Copyright ( 2011 You-Jiang Liu et al. This is an open access article distributed under the Creative Commons Attribution License, which permits unrestricted use, distribution, and reproduction in any medium, provided the original work is properly cited.

\begin{abstract}
We propose a two-step identification approach for twin-box model (Wiener or Hammerstein) of RF power amplifier. The linear filter block and the static nonlinearity block are extracted, respectively, based on least-squares method, by iterative calculation. Simulations show that the method can get quite accurate parameters to model different nonlinear models with memory such as Wiener, Hammerstein, Wiener-Hammerstein (W-H), and memory polynomial models, hence, demonstrating its robustness. Furthermore, experimental results show excellent agreement between measured output and modeled output, where one carrier WCDMA signal is used as the excitation for a wideband RF amplifier.
\end{abstract}

\section{Introduction}

New signal modulation formats in modern communication systems are with high peak to average ratio (PAR) and wide bandwidth. Power amplifiers (PAs) excited by such signals exhibit different nonlinearity and memory effects compared with the case of single-tone excitation. Consequently, the development of behavioral models is indispensable for performance analysis of PAs and system simulation with PAs. The Volterra model [1] can be applied successfully to express PAs characteristics with memory effects, but with very complicated coefficients. With a reduction of the coefficients number, many simplified approximations for Volterra model are Wiener, Hammerstein, Wiener-Hammerstein (W-H), memory polynomial models [1-5]. Especially, the Wiener model and Hammerstein model, known as twin-box models, are the most popular ones. Usually, they can model PAs' behavior accurately with less complexity. The identification process for the twin-box models is faster than that of Volterra model also. Some previous works on twin-box models identification have been summarized or developed in [6-10]. Reference [6] summarizes different types of methods about Wiener model identification. In [7], the author proposes a new recursive identification method, based on the old one in [8]. In [9], it points out that Hammerstein model permits linear regression. However, identification of Wiener model is more complicated, where estimation of the intermediate variable is performed firstly, and then a two-step estimation of the Wiener coefficients by linear regression is available. The identification method in [10] is based on the artificial intelligence technique of swarm intelligence. In summary, most of these identification methods are either complicated or with low accuracy.

In this paper, we consider the twin-box models identification process and propose a novel identification method with simplicity. The model output is with a high degree of accuracy compared with the measured output. Different models such as Wiener, Hammerstein, Wiener-Hammerstein (W-H), and memory polynomial models can be modeled by either twin-box models (Wiener, or Hammerstein) with this identification method. As a further evaluation, measurement setup and experimental results for a wideband amplifier operating at $2.14 \mathrm{GHz}$ of one carrier WCDMA signal are presented. The results show high accuracy of the models.

\section{Twin-Box Models Identification}

The relationship between input and output complex envelopes of PAs can be described as Wiener model or 


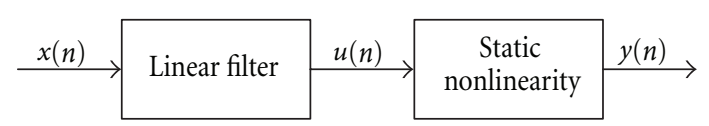

(a)

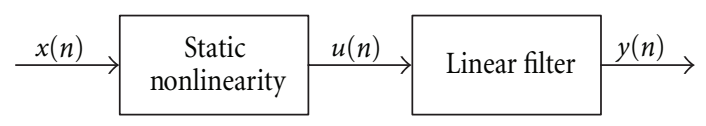

(b)

FIGURE 1: Block diagram of the twin-box models: (a) Wiener model and (b) Hammerstein model.

Hammerstein model, as shown in Figures 1(a) and 1(b). The Wiener is a cascade of a linear filter followed by a static nonlinearity, and it is the other way round-the Hammerstein is with a static nonlinearity followed by a linear filter. Usually, the linear filter can be approximately expressed by a finite impulse response (FIR) filter, and the static nonlinear block can be expressed as a polynomial form of finite order [11].

In our identification approach, the linear filter block and the static nonlinearity block are extracted, respectively, according to the collection of baseband input $\{x(n)\}$ and output $\{y(n)\}$ of PAs, both with sample length of $M$. We assume the FIR parameters for linear filter are $\mathbf{T}=$ $\left[t_{0}, t_{1}, t_{2}, \ldots, t_{Q}\right]^{\mathrm{T}}$, and the polynomial parameters for static nonlinear block are $\mathbf{A}=\left[a_{1}, a_{3}, a_{5}, \ldots, a_{2 N-1}\right]^{\mathrm{T}}$, where $Q$ and $2 N-1$ are memory depth and nonlinear order, respectively. Especially, we only consider the odd order nonlinearities of PAs because the even order components fall into the band far from the in-band signal and can be filtered easily as a result. Taking the identification process of Wiener model as an example, the identification procedure is as follows

(1) Collecting input and output samples: $\{x(n)\}$ and $\{y(n)\}$, with length of $M$.

(2) Initialization: $\mathbf{T}=[1,0,0, \ldots, 0]^{\mathrm{T}}, \mathbf{A}=$ $[1,0,0, \ldots, 0]^{\mathrm{T}}$.

(3) Step 1: identifying static nonlinear block. Let $\{x(n)\}$ pass through $\mathbf{T}$ to get $\{u(n)\}$, using $\{u(n)\}$ and $\{y(n)\}$ to extract A. Define the output vector of static nonlinear block as $\mathbf{y}=[y(1), y(2), \ldots, y(M)]^{\mathrm{T}}$ and the input matrix with $N$ order as

$$
\mathbf{U}=\left[\begin{array}{cccc}
u(1) & u^{3}(1) & \cdots & u^{2 N-1}(1) \\
u(2) & u^{3}(2) & \cdots & u^{2 N-1}(2) \\
\vdots & \vdots & \vdots & \vdots \\
u(M) & u^{3}(M) & \cdots & u^{2 N-1}(M)
\end{array}\right]
$$

At convergence, we should have

$$
\mathrm{y}=\mathrm{UA} \text {. }
$$

The least-squares solution for (2) is

$$
\mathbf{A}=\left(\mathbf{U}^{\mathrm{H}} \mathbf{U}\right)^{-1} \mathbf{U}^{\mathrm{H}} \mathbf{y}
$$

where $(\cdot)^{\mathrm{H}}$ denotes the complex conjugate transpose.
(4) Step 2: identifying linear filter block. Let $\{y(n)\}$ pass through the inverse of $\mathbf{A}$ (the "inversion calculation process" to calculate intermediate variable of $u(n))$, to inversely estimate the input vector of static nonlinear block as $\mathbf{u}^{\prime}$. For the static nonlinearity block, there is $y(n)=$ $\sum_{k=1}^{N} A(k) u(n)|u(n)|^{2 k-2}$. Then we have $u(n)=[y(n)-$ $\left.\sum_{k=2}^{N} A(k) u(n)|u(n)|^{2 k-2}\right] / A(1)$. For the purpose of "inversion calculation", we firstly initialize $\mathbf{u}_{0}^{\prime}=\mathbf{y} / A(1)$ by ignoring the higher order nonlinear terms, then iteratively calculate the estimated input vector corresponding to $\mathbf{A}$ as

$$
\mathbf{u}_{i+1}^{\prime}=\frac{\left(\mathbf{y}-\sum_{k=2}^{N} A(k) \mathbf{u}_{i}^{\prime}\left|\mathbf{u}_{i}^{\prime}\right|^{2 k-2}\right)}{A(1)},
$$

where $A(k)$ is the $k$ th element of $\mathbf{A}$, and $\mathbf{u}_{i}^{\prime}$ is estimated input vector of static nonlinear block of the $i$ th iteration. Usually, the iteration process in (4) can be stopped after several iterations (e.g., 5 to 10 iterations). The selection of how long the iteration should be kept is not a crucial factor, because the external iteration of the proposed two steps in the whole identification algorithm can ensure the convergence of the process.

After that, we use $\{x(n)\}$ and $\mathbf{u}^{\prime}$ to extract T. Define the output vector of linear filter block with length of $L(Q<L \leq$ $M)$ as $\widehat{\mathbf{u}}=\left[\mathbf{u}^{\prime}(1), \mathbf{u}^{\prime}(2), \ldots, \mathbf{u}^{\prime}(L)\right]^{\mathrm{T}}$ and the input matrix with $Q$-depth as

$$
\mathbf{X}=\left[\begin{array}{cccc}
x(1) & 0 & \cdots & 0 \\
x(2) & x(1) & \cdots & 0 \\
\vdots & \vdots & \cdots & \vdots \\
x(L) & x(L-1) & \cdots & x(L-Q)
\end{array}\right]
$$

similarly as the solution of static nonlinear block, $\mathbf{T}$ can be expressed as

$$
\mathbf{T}=\left(\mathbf{X}^{\mathrm{H}} \mathbf{X}\right)^{-1} \mathbf{X}^{\mathrm{H}} \widehat{\mathbf{u}}
$$

(5) Going back to (3) and (4) to iteratively extract the coefficients of $\mathbf{T}$ and $\mathbf{A}$.

This identification method is with high accuracy and fast convergence speed. In most cases, it can converge within two iterations. A similar approach to identify Hammerstein model can be deduced. Noticeably, for Hammerstein model identification, the static nonlinear block should be identified firstly and then followed by the linear filter identification process during the iterations.

\section{Simulations for Different PA Models}

In order to evaluate the performance of the proposed twostep identification approach, we apply the method to different nonlinear models with memory through computer simulations. The Wiener, Hammerstein, Wiener-Hammerstein (W-H), and memory polynomial models PAs are considered as the PAs we want to identify. The coefficients of these PAs are set as practical-like ones. The coefficients of them are 
TABLE 1: PAs coefficients used for simulation.

\begin{tabular}{ll}
\hline Wiener PA & $H(z)=1+0.1 z^{-1}+0.3 z^{-2}$ \\
& $b_{1}=1.0108+0.0858 j, b_{3}=0.0879-0.0583 j$, \\
& $b_{5}=-0.1392-0.0989 j$ \\
\hline \multirow{3}{*}{ Hammerstein PA } & $b_{1}=1.1190+0.0837 j, b_{3}=0.1219-0.1513 j$, \\
& $b_{5}=-0.5122-0.3105 j$ \\
& $H(z)=1+0.5 z^{-2}$ \\
& $H_{1}(z)=\left(1+0.1 z^{-1}+0.3 z^{-2}\right) /\left(1-0.2 z^{-1}\right)$ \\
W-H PA & $b_{1}=1.0108+0.0858 j, b_{3}=0.0879-0.0583 j$, \\
& $b_{5}=-0.1392-0.0989 j$ \\
& $H_{2}(z)=\left(1-0.1 z^{-2}\right) /\left(1-0.1 z^{-1}\right)$ \\
\hline Mem. poly. PA & $b_{10}=1.4513+0.932 j, b_{11}=-0.123-0.023 j$, \\
& $b_{12}=0.152-0.230 j$, \\
& $b_{30}=-0.332-0.430 j, b_{31}=0.322+0.243 j$, \\
& $b_{32}=-0.123-0.120 j$, \\
& $b_{50}=-0.255-0.254 j, b_{51}=-0.0213-0.411 j$, \\
& $b_{52}=0.0233+0.233 j$. \\
\hline
\end{tabular}

listed in Table 1, where $H(z)$ and $b_{i}$ are the FIR coefficients and polynomial coefficients, respectively. For memory polynomial models, $b_{k q}$ are coefficients with $k$ representing nonlinear order and $q$ representing memory depth.

We extract the modeling parameters of twin-box models through 64QAM with $8 \times$ sampling rate firstly. Then the models are validated by using a different type signal of one carrier WCDMA with $10 \times$ sampling rate. Normalized mean square error (NMSE) is used to evaluate the modeling accuracy, which is defined as

$$
\mathrm{NMSE}=10 \log _{10} \frac{\sum_{n}\left|y(n)-y_{\text {model }}(n)\right|^{2}}{\sum_{n}|y(n)|^{2}} .
$$

The results of one carrier WCDMA validation for different PAs identified as either Wiener model or Hammerstein model are summarized in Table 2. For all test cases, we select memory depth of $Q=2$ and polynomial order of $2 N-1=5$, resulting in the total number of model parameters to be 6 . It is obvious that Wiener modeling exhibits better performances than Hammerstein modeling in general. In particular, both twin-box models can model the same model PAs of themselves with a very high degree of accuracy, which are $-289.2 \mathrm{~dB}$ NMSE with Wiener modeling for Wiener PA, and -92.1 dB NMSE with Hammerstein modeling for Hammerstein PA. Noticeably, an NMSE of $-289.2 \mathrm{~dB}$ with Wiener modeling for Wiener PA is just obtained in simulation. In real world, it is impossible to get such accuracy, because a real PA does not really have its model, and the model is just an approximation for it. Besides, we can see that NMSE of the Hammerstein model from Hammerstein virtual measurement data is not so good as is, for example, the NMSE of the identified Wiener model starting from Wiener virtual measurement data. The reason is that, in the Hammerstein model identification process, iterations of the "inversion calculation process" (similar as (4)) does not last too long (10 iterations here). If the "inversion calculation process" is performed with sufficient iterations (e.g., 50 iterations), NMSE of modeling would be

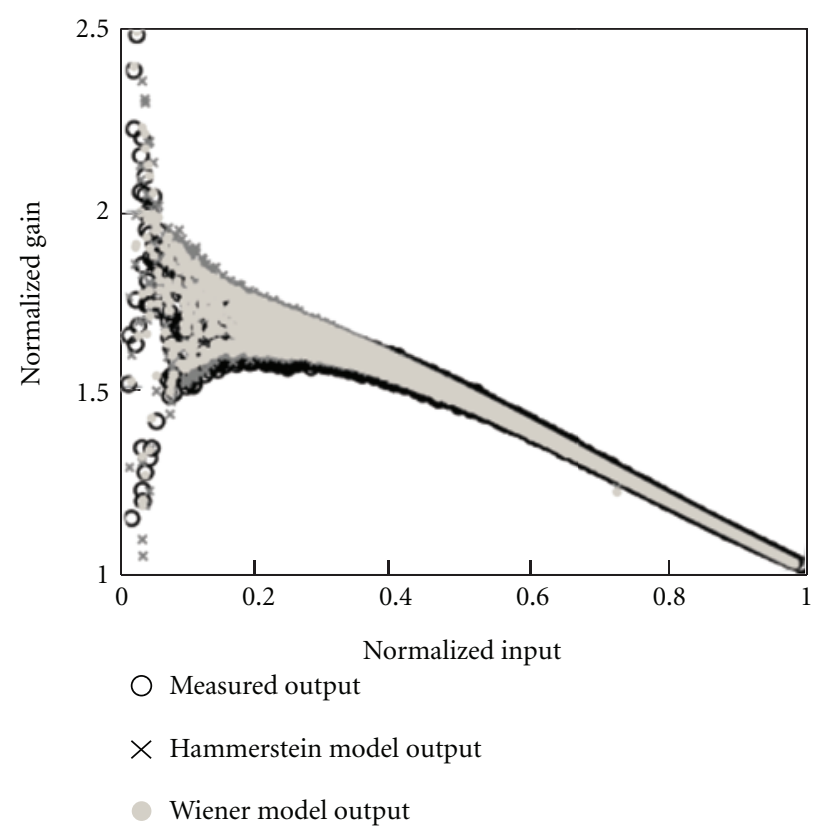

(a)

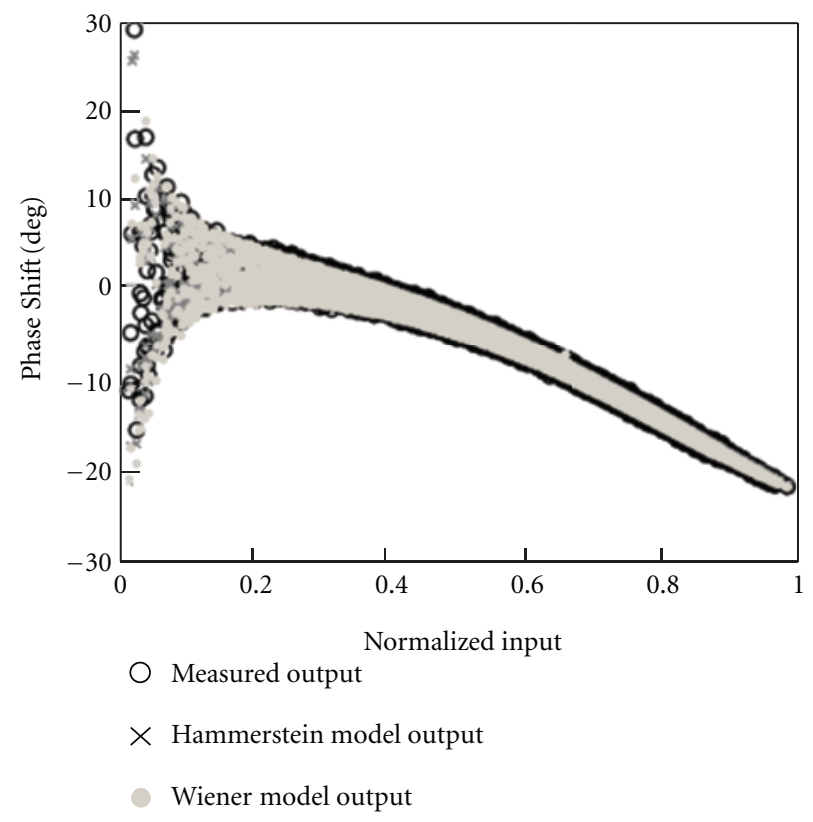

(b)

FIgURE 2: Measured and modeled characteristics of the RF amplifier: (a) AM/AM and (b) AM/PM.

$-292.8 \mathrm{~dB}$ rather than $-92.1 \mathrm{~dB}$. For comparison, Table 2 also gives the results of Wiener modeling method in [9]. The results show the superiority of the proposed method, with higher modeling accuracy.

\section{Experimental Results}

During the experimental validation process, an extensively used test bed is employed for measurement purpose, which is based on an arbitrary waveform generator and a vector signal 
TABLE 2: Modeling performances of twin-box models for different PAs with one carrier WCDMA excitation.

\begin{tabular}{lccc}
\hline & NMSE [dB] of Wiener modeling & NMSE [dB] of Hammerstein modeling & $\begin{array}{c}\text { NMSE [dB] of Wiener modeling with } \\
\text { the method in [9] }\end{array}$ \\
\hline Wiener PA & -289.2 & -49.1 & -40.9 \\
Hammerstein PA & -48.0 & -92.1 & -39.8 \\
W-H PA & -49.1 & -39.6 & -39.1 \\
Mem. poly. PA & -40.4 & -40.0 & -34.7 \\
\hline
\end{tabular}

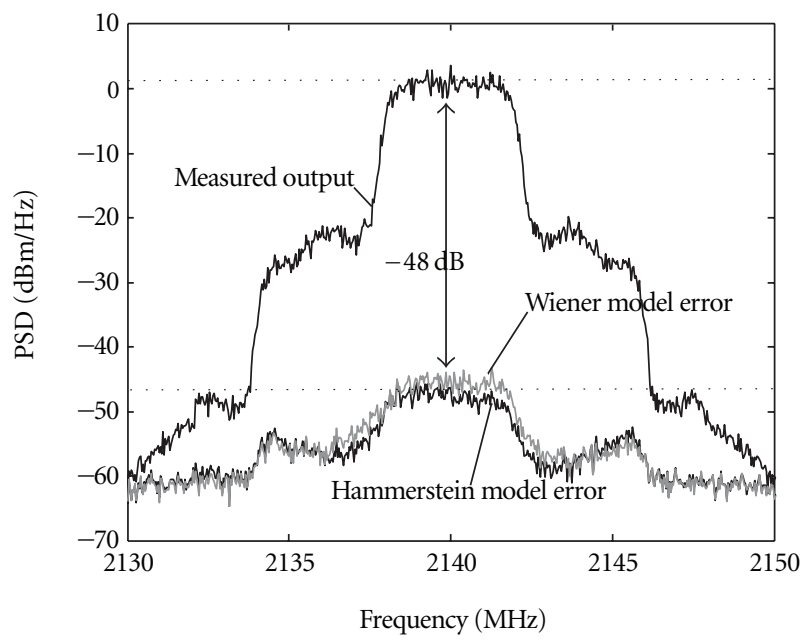

FIGURE 3: Measured and modeled spectra of the RF power amplifier.

analyzer. The baseband $10 \times$ sampled WCDMA signal with $5 \mathrm{MHz}$ bandwidth is uploaded into the arbitrary waveform generator and upconverted to $2.14 \mathrm{GHz}$ to construct the real world RF signal. Then, the signal is transferred to a wideband RF amplifier, which operates from $100 \mathrm{kHz}$ to $3 \mathrm{GHz}$ with $30 \mathrm{~dB}$ gain and $1 \mathrm{~W}$ saturated output power. Finally, the output of the amplifier is captured from the vector signal analyzer to acquire the baseband complex-envelope samples. The input of the amplifier can also be acquired through the same way to get baseband samples. Notably, the input signal should be sampled with the same sampling rate like that of sampling the output signal. This creates a one-to-one relationship between the input and output samples. And we capture 49800 samples both for input and output. During the models training procedures, some 10000 samples are used to extract the models parameters. Then the models are verified at every sampling time with all the 49800 samples.

Figure 2 shows the measured and modeled AM/AM and $\mathrm{AM} / \mathrm{PM}$ of the RF amplifier when it operates at $-6 \mathrm{dBm}$ average input level where it has been pushed to its saturation point beyond the $1 \mathrm{~dB}$ compression point ( at about $-8 \mathrm{dBm}$ input). The results show that both Wiener model and Hammerstein model can express the amplifier's AM/AM and $\mathrm{AM} / \mathrm{PM}$ very well, where the memory depth of $Q=2$ and polynomial order of $2 N-1=5$ are selected. From the compression and dispersion of the curves, we can see the amplifier has been driven to exhibit strong nonlinearity and weak memory effects. With respect to the modeling accuracy,
-45.1 dB NMSE with Wiener modeling and -46.6 dB NMSE with Hammerstein modeling are achieved, respectively.

The normalized measured and modeled spectra for the amplifier are depicted in Figure 3, which also show excellent agreement of the twin-box models with measurement result. The model errors of in-band for both twin-box models are about $-48 \mathrm{~dB}$ lower than measured output. Besides, the outof-band performance can also be modeled very well.

\section{Conclusions}

The twin-box models have been extensively used in power amplifier modeling with memory effects and in digital predistortion linearization technique. The novel two-step identification approach has been validated through $5 \mathrm{MHz}$ WCDMA signal excitation. Simulations show very small modeling error in the order of $-40 \mathrm{~dB} \sim-50 \mathrm{~dB}$ for different kind of power amplifiers with memory. Thus, the robustness with the twin-box models has been demonstrated. Experimental results also show excellent agreements between the measured output and model output. As a further application of the twin-box modeling method, the most cost-effective linearization technique of digital predistortion for power amplifier can be easily employed by an indirect learning architecture.

\section{References}

[1] D. Schreurs, M. O’Droma, A. A. Goacher, and M. Gadringer, RF Power Amplifier Behavioral Modeling, Cambridge University Press, New York, NY, USA, 2008.

[2] H. Ku and J. S. Kenney, "Behavioral modeling of nonlinear RF power amplifiers considering memory effects," IEEE Transactions on Microwave Theory and Techniques, vol. 51, no. 12, pp. 2495-2504, 2003.

[3] M. Isaksson, D. Wisell, and D. Rönnow, "A comparative analysis of behavioral models for RF power amplifiers," IEEE Transactions on Microwave Theory and Techniques, vol. 54, no. 1, pp. 348-359, 2006.

[4] F. M. Ghannouchi and O. Hammi, "Behavioral modeling and predistortion," IEEE Microwave Magazine, vol. 10, no. 7, pp. 53-64, 2009.

[5] P. N. Landin, M. Isaksson, and P. Händel, "Parameter extraction and performance evaluation method for increased performance in RF power amplifier behavioral modeling," International Journal of RF and Microwave Computer-Aided Engineering, vol. 20, no. 2, pp. 200-208, 2010.

[6] T. Ogunfunmi, Adaptive Nonlinear System Identification: The Volterra and Wiener Model Approaches, Springer, New York, NY, USA, 2007. 
[7] F. Guo, A new identification method for Wiener and Hammerstein Systems, Doctor thesis, Universität Karlsruhe, Berlin, Germany, 2004.

[8] L. Ljung, "Convergence analysis of parametric identification methods," IEEE Transactions on Automatic Control, vol. 23, no. 5, pp. 770-783, 1978.

[9] P. L. Gilabert, G. Montoro, and E. Bertran, "On the wiener and hammerstein models for power amplifier predistortion," in Proceedings of the Asia-Pacific Microwave Conference, pp. 14, 2005.

[10] J. Liu, W. Xu, and J. Sun, "Nonlinear system identification of hammerstein and wiener model using swarm intelligence," in Proceedings of the IEEE International Conference on Information Acquisition '06, pp. 1219-1223, 2006.

[11] H. W. Kang, Y. S. Cho, and D. H. Youn, "Adaptive precompensation of wiener systems," IEEE Transactions on Signal Processing, vol. 46, no. 10, pp. 2825-2829, 1998. 

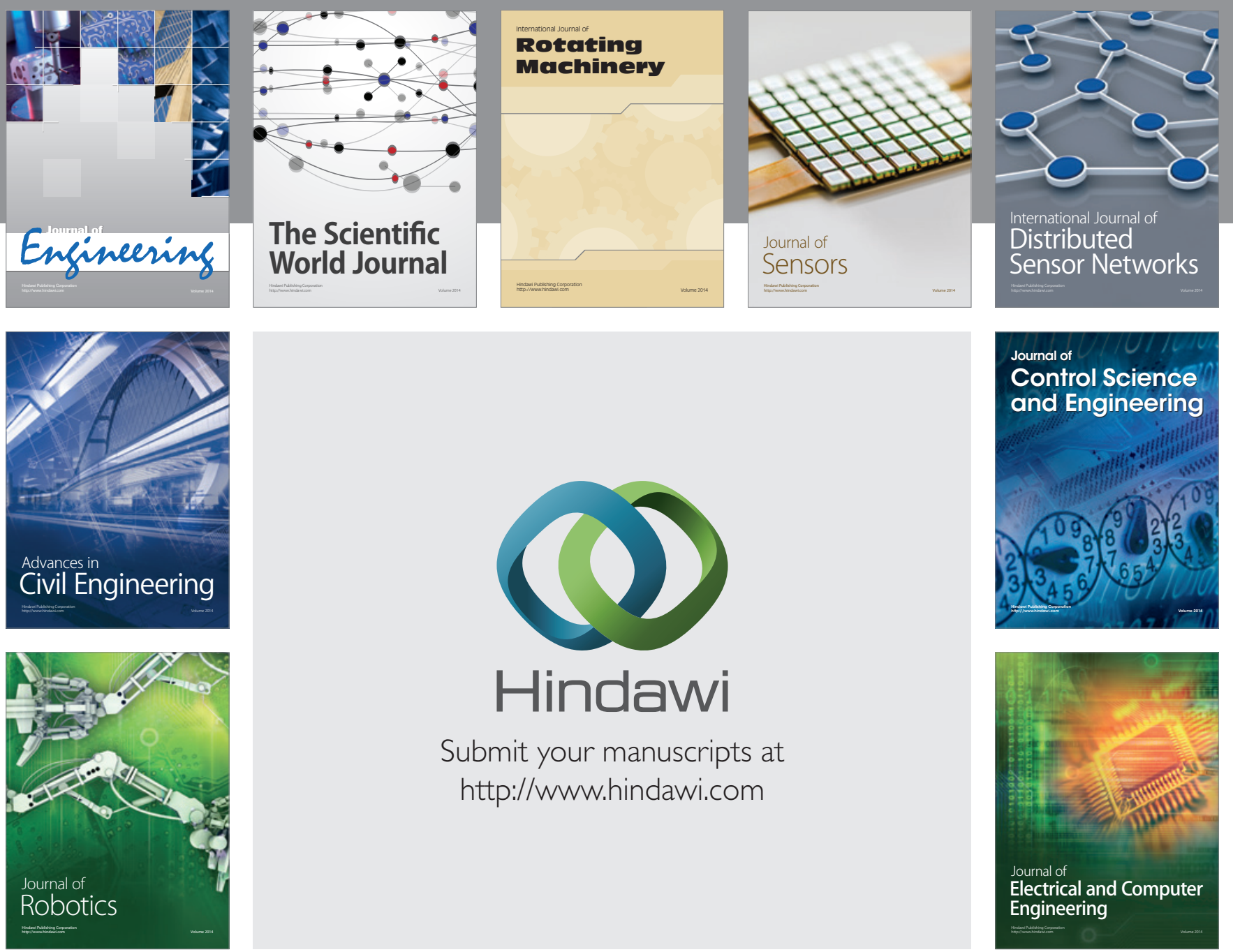

Submit your manuscripts at

http://www.hindawi.com
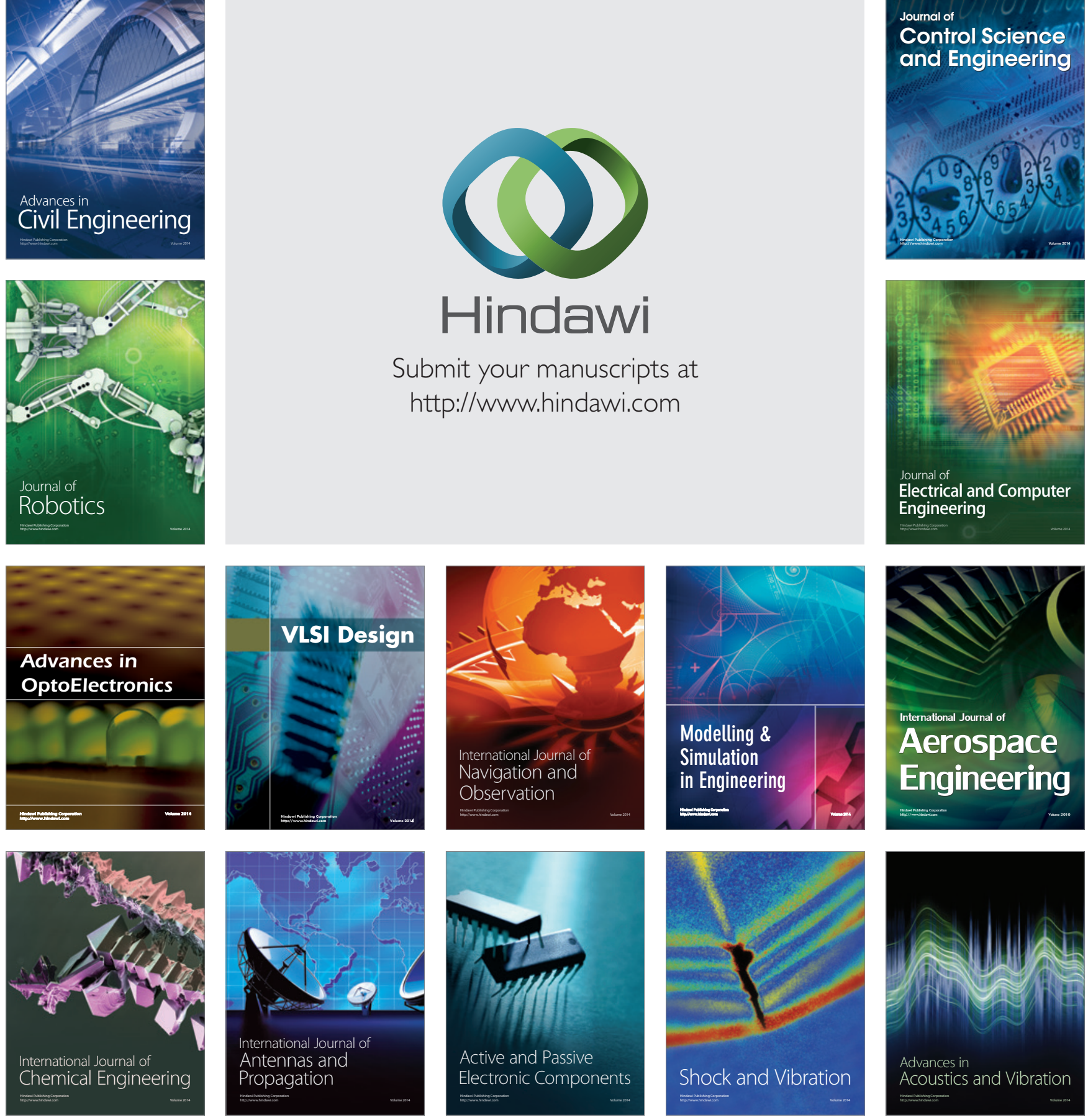